\title{
Erratum to: Type-4 spinors: transmuting from Elko to single-helicity spinors
}

\author{
C. H. Coronado Villalobos ${ }^{1, a}$, R. J. Bueno Rogerio ${ }^{2,3, b}$, E. F. M. T. São Sabbas ${ }^{1, c}$ \\ ${ }^{1}$ Instituto Nacional de Pesquisas Espaciais (INPE), São José dos Campos, SP 12227-010, Brazil \\ ${ }^{2}$ Instituto de Física e Química, Universidade Federal de Itajubá - IFQ/UNIFEI, Av. BPS 1303, Itajubá, MG 37500-903, Brazil \\ ${ }^{3}$ Departamento de Física e Química, Faculdade de Engenharia, Guaratinguetá, Universidade Estadual Paulista (UNESP), Guaratinguetá, \\ SP 12516-410, Brazil
}

Received: 23 May 2019 / Accepted: 2 July 2019 / Published online: 11 July 2019

(c) The Author(s) 2019

\section{Erratum to: Eur. Phys. J. C (2019) 79:308 https://doi.org/10.1140/epjc/s10052-019-6832-2}

The last co-Author's name in the original manuscript is not correct. In Sect. 3 of the original article, the Lorentz boost parameter presented in Eqs. (15) and (18), which refers to $\psi_{2 \downarrow}(\boldsymbol{p})$ and $\psi_{3 \downarrow}(\boldsymbol{p})$, must to be replaced. The correct form is

$\psi_{2 \downarrow}(\boldsymbol{p})=\sqrt{m}\left(\begin{array}{c}-i \mathcal{B}^{-}(p) \sin (\theta / 2) e^{-i \phi / 2} \\ i \mathcal{B}^{-}(p) \cos (\theta / 2) e^{i \phi / 2} \\ -\kappa_{2} \mathcal{B}^{+}(p) \sin (\theta / 2) e^{-i \phi / 2} \\ \kappa_{2} \mathcal{B}^{+}(p) \cos (\theta / 2) e^{i \phi / 2}\end{array}\right)$.

and

$\psi_{3 \downarrow}(\boldsymbol{p})=\sqrt{m}\left(\begin{array}{c}i \kappa_{3} \mathcal{B}^{-}(p) \sin (\theta / 2) e^{-i \phi / 2} \\ -i \kappa_{3} \mathcal{B}^{-}(p) \cos (\theta / 2) e^{i \phi / 2} \\ -\mathcal{B}^{+}(p) \sin (\theta / 2) e^{-i \phi / 2} \\ \mathcal{B}^{+}(p) \cos (\theta / 2) e^{i \phi / 2}\end{array}\right)$.

After performing the above modification, Eqs. (26) and (27) in Sect. 5, now, are replaced by

$$
\sum_{i=1}^{4} \psi_{i}(\boldsymbol{p}) \bar{\psi}_{i}(\boldsymbol{p})=2 \gamma_{\mu} p^{\mu}
$$

where the matrix $\gamma_{\mu} p^{\mu}$ reads

$$
\begin{aligned}
& \gamma_{\mu} p^{\mu} \\
& =\left(\begin{array}{cccc}
0 & 0 & E+p \cos (\theta) & p \sin (\theta) e^{-i \phi} \\
0 & 0 & p \sin (\theta) e^{i \phi} & E-p \cos (\theta) \\
E-p \cos (\theta) & -p \sin (\theta) e^{-i \phi} & 0 & 0 \\
-p \sin (\theta) e^{i \phi} & E+p \cos (\theta) & 0 & 0
\end{array}\right),
\end{aligned}
$$

after Eq. (28) on the original article, the correct is to note that the spin sums is Lorentz invariant. The operator that appears on the right hand side in Eq. (3) does not annihilates type-4 spinors.

Open Access This article is distributed under the terms of the Creative Commons Attribution 4.0 International License (http://creativecomm ons.org/licenses/by/4.0/), which permits unrestricted use, distribution, and reproduction in any medium, provided you give appropriate credit to the original author(s) and the source, provide a link to the Creative Commons license, and indicate if changes were made. Funded by SCOAP S $^{3}$

The original article can be found online at https://doi.org/10.1140/ epjc/s10052-019-6832-2.

\footnotetext{
a e-mail: carlos.coronado@inpe.br

b e-mail: rodolforogerio@unifei.edu.br

c e-mail: eliahfersaosabbas@gmail.com
} 